from life-threatening conditions to chronic relapsing long-term diseases as a result of significant advances in immunosuppressive therapy. Structured clinical assessment using Vasculitis Damage Index (DVI) should form the basis of a treatment plan and be used to document progress.

Objectives: To investigate the Vasculitis Damage Index and clinical manifestations in localized and systemic granulomatosis with poliangiitis in Mexican patients

Methods: We enrolled 61 patients with GPA according to The American College of Rheumatology (ACR) criteria at a referral hospital during the period from 2005 to 2015. Clinical and laboratory data, organ involvement and the Vasculitis Damage Index (VDI) were recorded at baseline. Patients were divide into systemic and localized form for their analysis.

Results: They were 61 GPA (34 men and 27 women) mean age 42 years old at diagnosis. Systemic form was observed in 53\% and localized form $47 \%$. Chronic sinusitis was the most frequent manifestation in $33 \%$ followed by otologic in $26 \%$. Subglottic stenosis 4 patients, alveolar hemorrhage $1 \%$. Of the patients with the systemic form 22 presented focal and segmental glomerulonephritis and 10 patients (32\%) rapidly progressive glomerulonephritis. Distal-symmetric polyneuropathy and cranial neuropathy were present in $24 \%$; scleritis $24.5 \%$ and proptosis in $18 \%$, palpable purpura $26.2 \%$ and ulcers in 9 patients (14.8\%). The VDI score in the systemic form was 3.8 and in the localized 2.6, $p=$ NS. The disease related damage was pronounced in kidneys and upper airways. The majority of patients in the induction to remission phase received steroids plus cyclophosphamide, 7 patients also received plasmapheresis and in maintenance phase they were treated with methotrexate or azathioprine.

Conclusions: In this cohort of patients with GPA, a high chronic damage was found which was similar in both systemic and localized forms of this vasculitis. The VDI was more prominent in kidneys and upper airways in GPA patients

References:

[1] Bhamra K, et al. Damage assessment in ANCA-associated vasculitis. Curr Rheumatol Rep. 2012; 14:494-50.

[2] Kamali S, et al. Predictors of damage and survival in patients with Wegener's granulomatosis: analysis of 50 patients, J Rheumatol 2010;37:374-78.

[3] Luqmani $R$, et al. State of the art in the treatment of systemic vasculitides. Front Immunol 2014;5:471.

Disclosure of Interest: None declared

DOI: 10.1136/annrheumdis-2017-eular.5690

\section{AB0586 EVALUATION OF ASSSOCIATION BETWEEN ANTIPHOSPHOLIPID ANTIBODIES OR LUPUS ANTICOAGULANT POSITIVITY AND SEVERITY OF VASCULAR INVOLVEMENT IN TAKAYASU ARTERITIS PATIENTS}

E. Firat ${ }^{1}$, A. Erden ${ }^{2}$, A. Sari ${ }^{2}$, B. Armagan ${ }^{2}$, L. Kilic ${ }^{2}$, O. Karadag ${ }^{2}$, A. Akdogan ${ }^{2}{ }^{1}$ Internal Medicine, Hacettepe University Faculty of Medicine;

${ }^{2}$ Rheumatology, Hacettepe University of Medicine, Ankara, Turkey

Background: Takayasu Arteritis is a rare large-vessel vasculitis variant that affects the aorta and its main branches and the pulmonary arteries. Antiphospholipid syndrome is characterized by obstetric and thrombotic complications in the presence of antiphospholipid antibodies, which consist of anticardiolipin antibody, lupus anticoagulant and anti $-\beta 2$ glycoprotein I. The association of antiphospholipid antibodies and Takayasu arteritis is very rare and few cases decumented it, while others argued aganist such association.

Objectives: This study was planned to find out the prevelance of immunoglobulinIgM/G anti-cardiolipinantibodies, anti beta 2 glycoprotein-1 1 antibodies and lupus anticogulant and evaluate the relationship between these antibodies and disease severity/complications in Takayasu arteritis patients.

Methods: 53 patients with Takayasu arteritis patients were enrolled in this study. We obtained blood samples to detectlgM/G anti-cardiolipin antibodies, anti beta 2 glycoprotein 1 antibodies and lupus anticogulant (LA) levels from all patients during their routine control. ImmunoglobulinlgM/G anti-cardiolipin antibody, anti beta 2 glycoprotein 1 antibodies were measured by using a standardized enzymelinked immunosorbent assay (ELISA) and lupus anticogulant was measured using the diluteRussell's viper venom time (dRVVT).

Results: No patients was positive forlgM/G anti-cardiolipin antibody. Seven were positive immunoglobulin IgM anti beta 2 glycoprotein 1 antibodies, three were positive immunoglobulin IgG anti beta 2 glycoprotein 1 antibodies, one waspositive LA. All of the antibody titters were low. TA patients who had antibody positivity had longer disease duration $(p<0.05)$. Antibody and LA positive patients had superior mesenteric artery and celiac artery involvement more frequently then the antibody negative patients $(p<0.05)$.

Conclusions: In this study that there were no association between the antibodies positivity and vascular involvement or disease complications and severity in TA patients. In conclusion we can not suggest the routine evaluation of antiphospholipid antibodies or lupus anticoagulant test during the follow-upTakayasu arteritis patients. These antibodies may only be measured in the presence of clinical suspicion.

Disclosure of Interest: None declared

DOI: 10.1136/annrheumdis-2017-eular.3529

\section{AB0587 ANTINEUTROPHILIC CYTOPLASMIC ANTIBODY-ASSOCIATED VASCULITIS AND HYPOCOMPLEMENTEMIA: CLINICAL IMPACT AND OUTCOME}

S. Deshayes ${ }^{1}$, A. Aouba ${ }^{1}$, K. Khoy ${ }^{2}$, D. Mariotte ${ }^{2}$, T. Lobbedez ${ }^{3}$, N. Martin

Silva ${ }^{1} .{ }^{1}$ Department of Internal Medicine; ${ }^{2}$ Department of Immunology;

${ }^{3}$ Department of Nephrology, CHU Côte de Nacre, Caen, France

Background: Although their pathophysiology are still largely unknown, there are growing evidences that complement $(C)$ alternative pathway activation is implicated in antineutrophilic cytoplasmic antibody-associated vasculitis (AAV) pathogenesis.

Objectives: The aim of our study was to evaluate the clinical characteristics and outcome of AAV patients, according to their serum $\mathrm{C}$ levels at diagnosis.

Methods: A retrospective monocentric study carried out in Caen University Hospital led to identify proteinase-3 (PR3) or myeloperoxidase (MPO)-ANCA AAV patients (via an ELISA technique). All patients with available C3 and C4 levels (by nephelemetry) at diagnosis were included, except for eosinophilic granulomatosis with polyangiitis (EGPA), which has a different pathophysiology. AAV were classified between granulomatosis with polyangiitis (GPA) or microscopic polyangiitis (MPA), and limited or severe forms according to respectively European Medicines Agency vasculitis algorithm and WGET group. Patients were categorized in the hypocomplementemia group if the C3 or C4 level at diagnosis was below the lower limit of the normal range (respectively $750-1400 \mathrm{mg} / \mathrm{l}$ and $100-340 \mathrm{mg} / \mathrm{l}$ ). Categorical variables were reported as percentages and compared using Fisher's tests. Continuous variables were expressed as means and analyzed using Student's t-test. Associations between survival, renal survival and relapse-free survival, and low serum C levels were evaluated by the log-rank test. A p-value $<0.05$ was considered to be statistically significant.

Results: Among the 157 AAV patients identified, 81 were excluded (8 EGPA, 73 without $\mathrm{C} 3$ and $\mathrm{C} 4$ determinations before treatment initiation). On the 76 AAV included (43 GPA, $33 \mathrm{MPA}$ ), median age at diagnosis was 65 years (M/F, 38/38). Clinical presentations included constitutional symptoms (56, 73.7\%), pulmonary $(52,68.4 \%)$, renal $(50,65.8 \%)$, rheumatologic $(43,56.6 \%)$, and ear, nose or throat $(37,48.7 \%)$ involvements, without statistical differences between groups. Twelve $(15.8 \%)$ deaths and 41 relapses in $25(32.9 \%)$ patients were noted (median follow-up: 38 months). Four patients (5.3\%) had hypocomplementemia: 1 patient had isolated low C3 level, 1 had isolated low $\mathrm{C} 4$ level, and 2 had both low $\mathrm{C}$ levels. All 4 patients had renal involvement. The $C$ level, controlled in 1 patient, became normal 1 month later. No thrombotic microangiopathy (TMA) features were found on the 2 performed kidney biopsies.

\begin{tabular}{lccc}
\hline & $\begin{array}{c}\text { Hypocomplementemia } \\
\text { group } \\
(\mathrm{n}=4)\end{array}$ & $\begin{array}{c}\text { Normal complement } \\
\text { level group } \\
(\mathrm{n}=72)\end{array}$ & $\mathrm{p}$ value \\
\hline Male \% & 50 & 50 & 1 \\
Age at diagnosis (years), median \pm SD & $71 \pm 9$ & $65 \pm 16$ & 0.18 \\
BVAS, median \pm SD & $21 \pm 8$ & $18 \pm 7$ & 0.26 \\
Granulomatosis with polyangiitis \% & 75 & 57 & 0.64 \\
PR3-ANCA \% & 50 & 60 & 1 \\
Limited vasculitis $\%$ & 0 & 28 & 0.57 \\
End-stage renal disease \% & 75 & 15 & 0.02 \\
Death \% & 50 & 14 & 0.12 \\
Relapse \% & 25 & 36 & 1 \\
\hline
\end{tabular}

Survival and renal survival were significantly lower in the hypocomplementemia group ( $p=0.0011$ and $p<0.001$, respectively), but relapse-free survival was similar $(\mathrm{p}=0.1)$.

Conclusions: Hypocomplementemia at AAV diagnosis may be responsible for worse survival and renal prognosis. This particular phenotype may confer resistance to common immunosuppressive approaches as in thrombotic microangiopathy caused by abnormalities in the regulation of the $C$ system. These results also argue for larger studies and for investigating $\mathrm{C}$ pathway targeting.

Disclosure of Interest: None declared

DOI: 10.1136/annrheumdis-2017-eular.1293

\section{AB0588 CONCOMITANT ASSOCIATION OF GIANT CELL ARTERITIS AND MALIGNANCY: A MULTICENTER RETROSPECTIVE CASE-CONTROL STUDY}

S. Deshayes $^{1}$, N. Chanson ${ }^{2}$, K. Sacré ${ }^{2}$, C. Blanchard-Delaunay ${ }^{3}$, O. Espitia ${ }^{4}$, T. Le Gallou ${ }^{5}$, M. Groh ${ }^{6}$, J.-E. Kahn ${ }^{7}$, V. Grobost ${ }^{8}$, S. Humbert ${ }^{9}$, M. Samson ${ }^{10}$ R. Mourot Cottet ${ }^{11}, \mathrm{~K}$. Mazodier ${ }^{12}, \mathrm{~A}$. Dartevel ${ }^{13}, \mathrm{M}$. Versini ${ }^{14}, \mathrm{~A}$. Dumont ${ }^{1}$, B. Bienvenu ${ }^{1}, A$. Aouba ${ }^{1}, \mathrm{H}$. de Boysson ${ }^{1}$ on behalf of French Study Group for Large Vessel Vasculitis (GEFA). ${ }^{1}$ Department of Internal Medicine, CHU Côte de Nacre, Caen; ${ }^{2}$ Department of Internal Medicine, Hôpital Bichat, Paris;

${ }^{3}$ Department of Internal Medicine, Centre Hospitalier de Niort, Niort;

${ }^{4}$ Department of Internal Medicine, CHU Hotel Dieu, Nantes; ${ }^{5}$ Internal Medicine, CHU Rennes, Rennes; ${ }^{6}$ Department of Internal Medicine, Hôpital Cochin, Paris, ${ }^{7}$ Department of Internal Medicine, Hôpital Foch, Suresnes; ${ }^{8}$ Department of Internal Medicine, CHU Estaing, Clermont-Ferrand; ${ }^{9}$ Department of Internal Medicine, CHU de Besançon, Besançon; ${ }^{10}$ Department of Internal Medicine, Hôpital François-Mitterrand, Dijon; ${ }^{11}$ Department of Internal Medicine, Hôpital Civil, Strasbourg; ${ }^{12}$ Department of Internal Medicine, Hôpital de la Conception, 
Marseille; ${ }^{13}$ Department of Internal Medicine, CHU Grenoble, Grenoble: ${ }^{14}$ Department of Internal Medicine, Hôpital Archet 1, Nice, France

Background: Giant cell arteritis (GCA) is a large-vessel vasculitis affecting elderly people, and most frequently women (sex-ratio of 2.3). Some studies suggest an increased risk of malignancies in GCA.

Objectives: We aimed to describe the clinical, paraclinical characteristics and outcomes of GCA patients with concomitant malignancy and compare them to a control group without malignancy.

Methods: Patients with a diagnosis of GCA and of solid neoplasm or malignant blood disease, within one year before or after the diagnosis of vasculitis, were included. A random group of age-matched (3:1) control patients from our monocentric inception cohort of GCA patients from Caen University Hospital was constituted.

Results: Twenty-four observations were collected (median age 75.5 years). All fulfilled $\geq 3 / 5$ ACR criteria. Temporal artery biopsy was positive in 17 cases $(70.8 \%)$. There were 1 active $(4.2 \%)$ and 9 former (37.5\%) smokers. Only 1 patient had a previous prostate cancer. Malignancies were 10 malignant blood diseases (41.7\%, 3 chronic lymphoid leukemias, 3 essential thrombocythemias, 1 myeloma, 1 chronic myelomonocytic leukemia, 1 MALT lymphoma, 1 Waldenström's macroglobulinemia) and 14 solid neoplasms (58.3\%, 3 lung, 3 breast, 2 prostate, 1 thyroid, 1 colon, 1 pleural cancers, 1 melanoma, 1 Kaposi's sarcoma and 1 Merkel cell carcinoma). Malignancy was diagnosed at a median of 1 month after GCA diagnosis in 21 patients and before in the other 3 . Diagnosis of malignancy was made in consultation in 5 patients ( 3 skin cancers and 2 breast cancers), on lab tests in 13 (thrombocytosis, anemia or increased prostate specific antigen) and on imaging in 6 . Treatments of malignancy included chemotherapy alone in 8 patients (33.3\%), simple monitoring in 6 patients $(25 \%)$, surgery alone in 4 patients $(16.7 \%)$, surgery and radiotherapy and/or chemotherapy in 4 patients $(16.7 \%)$, decrease of corticosteroids in 1 patient, and 1 patient was lost to follow-up. Two patients $(8.3 \%)$ died from infectious complications, 8 patients $(33.3 \%)$ had a GCA relapse, including one with concomitant malignancy relapse. After a median follow-up of 16 months [0-134], 5 patients $(20.8 \%)$ were weaned from steroids, all considered in malignancy remission. Seven patients $(29.1 \%)$ were still under chemotherapy, 9 patients (37.5\%) were considered to be in malignancy remission. There were more males in patients with concomitant malignancy, compared to the control group (respectively 15/24 and 21/72, $p<0.005$ ).

Conclusions: Our study shows an over-representation of male gender in GCA with concomitant malignancy. Vasculitis outcomes were not influenced by the malignancy treatment. The diversity of malignancies encountered in this study raises the issue of an incidental association. Initial clinical and paraclinical followup dictated by vasculitis may have led to an early identification of associated malignancy, and thus represent a lead time bias.

Disclosure of Interest: None declared

DOI: 10.1136/annrheumdis-2017-eular.1291

\section{AB0589 THE ROLE OF ANCA SPECIFICITY IN THE CLINICAL MANIFESTATIONS AT DISEASE ONSET: COMPARISON BETWEEN PATIENTS WITH GRANULOMATOSIS WITH POLYANGIITIS AND MICROSCOPIC POLYANGIITIS}

S. Monti ${ }^{1}$, M. Felicetti ${ }^{2,3}$, S. Balduzzi ${ }^{1}$, R. Padoan ${ }^{2}$, A. Berti ${ }^{3}$, G. Paolazzi $^{3}$, G. Brunori ${ }^{3}$, F. Schiavon ${ }^{2}$, R. Caporali ${ }^{1} .{ }^{1}$ Rheumatology, University of Pavia, IRCCS Policlinico San Matteo Foundation, Pavia; ${ }^{2}$ Rheumatology, Department of Medicine DIMED, University of Padova, Padova: ${ }^{3}$ Rheumatology, Santa Chiara Hospital, Trento, Italy

Background: ANCA specificity, rather than clinical diagnosis, has been suggested to influence the phenotype and clinical course of ANCA associated vasculitis (AAV) $(1,2)$.

Objectives: To investigate differences in clinical presentation at disease onset between MPO-ANCA-positive granulomatosis with polyangiitis patients (MPOGPA), PR3-ANCA-positive-GPA (PR3-GPA), and MPO-ANCA-positive microscopic polyangiitis (MPO-MPA).

Methods: Clinical records of AAV patients from three third level rheumatologic centers in Northern Italy were retrospectively analyzed.

Results: Of the 133 AAV patients included, 84 were PR3-GPA, 24 MPO-GPA, and 25 MPO-MPA. Patients with MPO-MPA were significantly older at diagnosis compared to both PR3-GPA and MPO-GPA (average age $63 \pm 10,49 \pm 15,55 \pm 29$, respectively) (Table 1 ).

Patients with MPO-GPA experienced a significant diagnostic delay compared to PR3-GPA ( $17 \pm 30$ vs $7 \pm 14, p=0.02)$. ENT involvement was equally frequent in both GPA groups despite ANCA specificity, and significantly more represented than the MPO-MPA group $(68 \%, 71 \%$ and $17 \%$ respectively; $p<0.001)$. Figure 1 . Renal involvement was significantly more frequent in MPO-MPA patients (100\%) compared to GPA $(p<0.001)$, without differences between MPO-GPA $(46 \%)$ and PR3-GPA (65\%). Alveolar haemorrhage (DAH) was an onset manifestation mainly in MPO-MPA compared to the other two groups (24\% vs $7 \%$ in PR3GPA; $p=0.02$ ). Cutenous manifestations, mainly purpura, were significantly more reported in PR3-GPA compared to MPO-MPA (29\% vs 4\%; $p=0.03$ ).

Conclusions: Clinical phenotype of GPA at disease onset did not seem to be influenced by ANCA specificity. Despite ANCA positivity (PR3 or MPO), GPA patients were significantly different from MPA.
Table 1. Clinical characteristics of patients with GPA and MPA at disease onset according to ANCA specificity

\begin{tabular}{|c|c|c|c|c|}
\hline & $\begin{array}{c}\text { GPA-PR3 (A) } \\
(n=84)\end{array}$ & $\begin{array}{c}\text { GPA-MPO (B) } \\
(n=24)\end{array}$ & $\begin{array}{c}\text { MPA-MPO (C) } \\
(n=25)\end{array}$ & $\mathrm{p}$ \\
\hline Male/female n (\%) & $46(55 \%) / 38(45 \%)$ & $10(42 \%) / 14(58 \%)$ & $10(40 \%) / 15(60 \%)$ & 0.29 \\
\hline $\begin{array}{l}\text { Age at disease onset } \\
\text { (average } \pm S D)\end{array}$ & $49 \pm 15$ & $55 \pm 19$ & $63 \pm 10$ & $\begin{array}{c}\quad p<0.001 \\
\text { A vs } C p<0.001 \\
\text { B vs } C p=0.03\end{array}$ \\
\hline $\begin{array}{l}\text { Diagnostic delay } \\
\text { (months } \pm \text { SD) }\end{array}$ & $7 \pm 14$ & $17 \pm 30$ & $10 \pm 19$ & 0.07 \\
\hline Systemic symptoms & $54(65 \%)$ & $16(67 \%)$ & $19(76 \%)$ & 0.59 \\
\hline ENT & $55(68 \%)$ & $17(71 \%)$ & $4(17 \%)$ & $<0.001$ \\
\hline Pulmonary & 57 (69\%) & $16(67 \%)$ & $16(64 \%)$ & 0.86 \\
\hline Cutaneous & $24(29 \%)$ & $5(21 \%)$ & $1(4 \%)$ & 0.03 \\
\hline Ocular & $16(20 \%)$ & $3(12 \%)$ & $2(8 \%)$ & 0.34 \\
\hline Cardiovascular & $4(5 \%)$ & $2(9 \%)$ & $1(4 \%)$ & 0.77 \\
\hline Gastrointestinal & $2(3 \%)$ & 0 & 0 & 0.56 \\
\hline Renal & $53(65 \%)$ & $11(46 \%)$ & $25(100 \%)$ & $<0.001$ \\
\hline Nervous system & $26(31 \%)$ & $8(33 \%)$ & $8(35 \%)$ & 0.94 \\
\hline
\end{tabular}

Figure 1. Differences in onset clinical manifestations among PR3-GPA, MPO-GPA and MPO-MPA

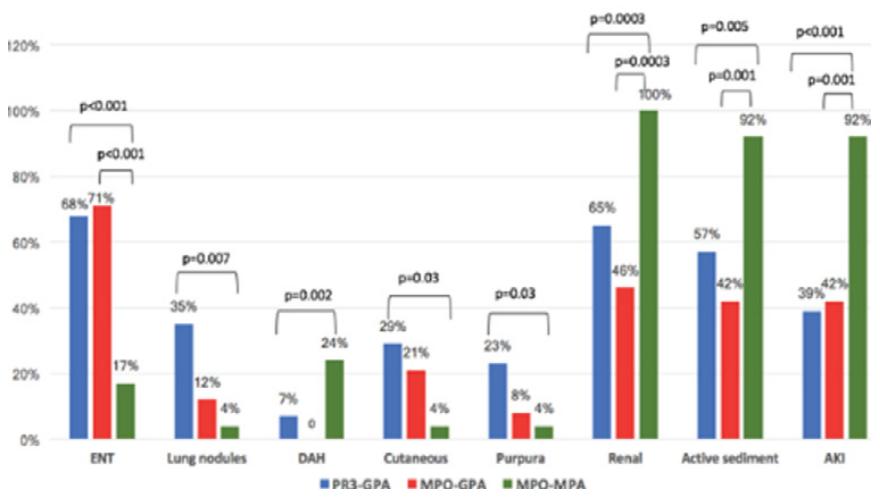

References:

[1] Schirmer JH, Wright MN, Herrmann K, et al. Myeloperoxidase-Antineutrophil Cytoplasmic Antibody (ANCA)-Positive Granulomatosis With Polyangiitis (Wegener's) Is a Clinically Distinct Subset of ANCA-Associated Vasculitis: A Retrospective Analysis of 315 Patients From a German Vasculitis Referral Center. Arthritis Rheumatol Hoboken NJ 2016;68:2953-63.

[2] Miloslavsky EM, Lu N, Unizony S, et al. Myeloperoxidase-Antineutrophil Cytoplasmic Antibody (ANCA)-Positive and ANCA-Negative Patients With Granulomatosis With Polyangiitis (Wegener's): Distinct Patient Subsets. Arthritis Rheumatol 2016;68:2945-2952.

Disclosure of Interest: None declared

DOI: 10.1136/annrheumdis-2017-eular.4760

\section{AB0590 PERFORMANCE OF 2017 ACR/EULAR PROVISIONAL CLASSIFICATION CRITERIA FOR GRANULOMATOSIS WITH POLYANGIITIS IN CHILEAN POPULATION}

S.F. Elgueta, L. Ricci Larrea, D. Vargas, P. Wurmann, I. Goecke. Hospital Clínico Universidad de Chile, Santiago, Chile

Background: Anca Associated Vasculitis (AAV), are a group of necrotizing primary vascultitis, whith multisistemic manifestation, of unknown etiology. The variants are: Microscopic Polyangiitis (MPA), Granulomatosis with polyangiitis (GPA), Granulomatosis whith Polyangiitis and Eosinophilia (GPE) and AAV limited to one organ. Until now, there are no diagnostic criteria for AAV. Therefore definitions, as Chapell Hill consensus Conference Nomenclature, classification criteria and the physician judgement are used for diagnosis. Currently the DCVAS (Diagnosis and Classification Criteria in Vasculitis) proyect is developing diagnostic criteria for AAV, using data-driven methods. The preliminary DCVAS classification criteria for granulomatosis whith polyangiitis has been recently realesead.

Objectives: To evaluate and compare the acuraccy of ACR/EULAR 2017 provisional Classification Criteria for GPA whith the ACR 1990 Classification Criteria In Chilean patients with AAV.

Methods: All adult patients ( $>18$ yo) with diagnoses of AAV according to their rheumatologist judgment, from 2000-2016 at the University of Chile, Clinical Hospital $(\mathrm{UCCH})$, were included. Clinical variables of interest were extracted form medical chart and AAV database, wich is kept for these patients at the Rheumatology Section of UCCH. Based on that data, the Classification criteria ACR 1990 and 2017preliminary ACR/EULAR (DCVAS) classification criteria for GPA were applied to each individual. Sensibility, especifity, Likehood ratio (LR $+/-$ ), predictive values (PPV/NPV) and accuracy were calculated for both sets of Criteria as compared to Clinical diagnosis

Results: 93 patient were included in the study. 59 patients with GPA, 33 with 